\title{
A Modified Functionally Generated Path Technique (FGP) for Making Posterior Unit Metal-Ceramic Crown
}

\author{
Saafi J*, Debbabi I, Alremthi HA, Hajjami H and Cherif M \\ Faculty of Dentistry, Avicenna Street Monastir, Tunisia
}

*Corresponding author: Saafi J, Faculty of Dentistry, Avicenna Street Monastir, Tunisia, Tel: +966549694345, E-mail: saafi_jilani@yahoo.fr

Citation: Saafi J, Debbabi I, Alremthi HA, Hajjami H, Cherif M (2018) A Modified Functionally Generated Path Technique(FGP) for Making Posterior Unit Metal-Ceramic Crown: A Case Report. J Oral Dent Sci 2: 103

Article history: Received: 11 October 2017, Accepted: 22 January 2018, Published: 24 January 2018

\section{Abstract}

Conventional techniques for construction accurate fixed prostheses have been unsuccessful in producing a prosthesis that can be inserted without intraoral occlusal adjustment. Successful oral rehabilitation requires precise fixed prostheses that are harmonious within occlusion and the stomatognathic system.

This article describes a new procedure to generate functional path occlusion for ceramometal unit crown.

Keywords: Functionally generated path (FGP); Temporary crown; Simple occlude; Unit crown

\section{Purpose}

In this study a Modified functionally generated path technique for unit crown was used as a new method of constructing of an accurate prosthesis that requires limited occlusal adjustment at the final seating. This article discusses the use of an alternative functionally generated path technique to fabricate a unit metal ceramic crown using a temporary crown and a simple plastic occluder rather than a twin stage one.

\section{Introduction}

The original technique was described by Meyer 73 years ago [1-3]. He termed it as the "chew-in" technique, and since then various researchers have refined the procedure. Over the years, the technique has been known by various names such as "functional bite technique", "generated path technique," and "cuspal tracing technique". Later, this technique was adapted for use in complete occlusal rehabilitation by Mann and Pankey [4-7]. It was also used for partial dentures, complete dentures, dental implant restorations and in the CAD/CAM techniques for full crown as Computer modeling of occlusal surfaces of posterior teeth $[2,3,8,9]$.

The functionally generated pathways (FGP) of occlusion refer to the registration of the paths of movement of the occlusal surfaces of the teeth of one dental arch, to the teeth or occlusion rims of the opposing arch, recorded three-dimensionally in the functional wax with the help of a plastic medium with acceptable condylar and anterior guidance and normal occlusal anatomy [10-12].

\section{Case presentation}

A 30-year healthy female patient presented to the department of fixed prosthodontics at the dental clinic of Monastir for restoring her maxillary right $2^{\text {nd }}$ premolar teeth. She also requested an esthetic and functional restoration. Patient's past dental history revealed a restoration of the second premolar, severely decayed due to dental caries 3 years ago, endodontically treated (the periapical X-ray is unfortunately not given in this article) and restored with casted post and core (Figure 1) [13].

Occlusion examination revealed presence of a group protection in lateral movements, and presence of anterior guidance.

All the teeth had a normal Morphology specially the teeth antagonists to this premolar. The patient was trained to close in maximum intercuspation position (MIP) and perform various eccentric movements (right lateral, left lateral and protrusive).

The tooth preparation for the abutment teeth, gingival retraction, final impression and master die preparation were done following the principles given by Schillingburg [14]. 


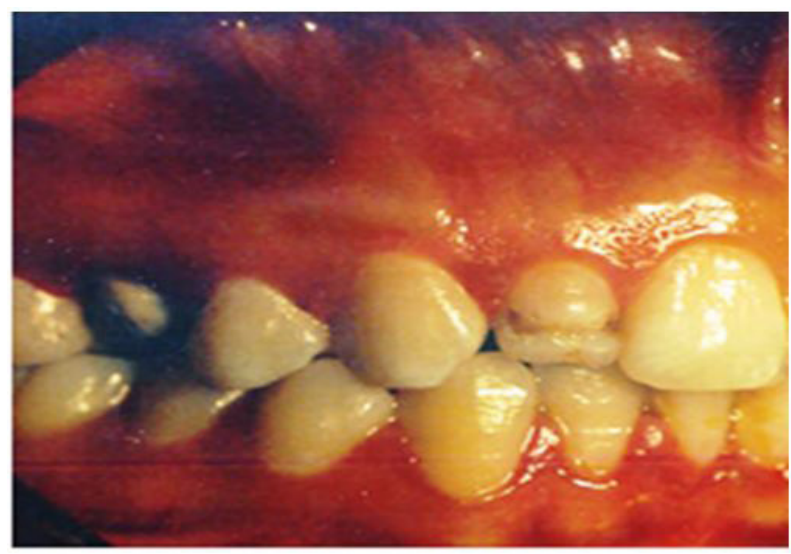

Figure 1: Prepared tooth $2^{\text {nd }}$ PM restored with core and post

A temporary acryl restoration has been carried on. After checking the occlusion contact, this provisional crown was reduced almost $1 \mathrm{~mm}$ in its occlusal surface.

After a layer of autopolymerizing resin (Duralay) was applied on the occlusion surface of the temporary restoration which was already put out of occlusion to achieve occlusal movement.

Before generating the occlusal morphology we ensured that temporary acryl restoration had adequate occlusal clearance and proper fit on the abutment.

The occlusal morphology was generated using pattern resin (GC Corporation, Tokyo, Japan) following the technique described by Dawson [15]. Pattern resin was mixed according to the manufacturer's instructions and applied on the occlusal surface (Figure 2).

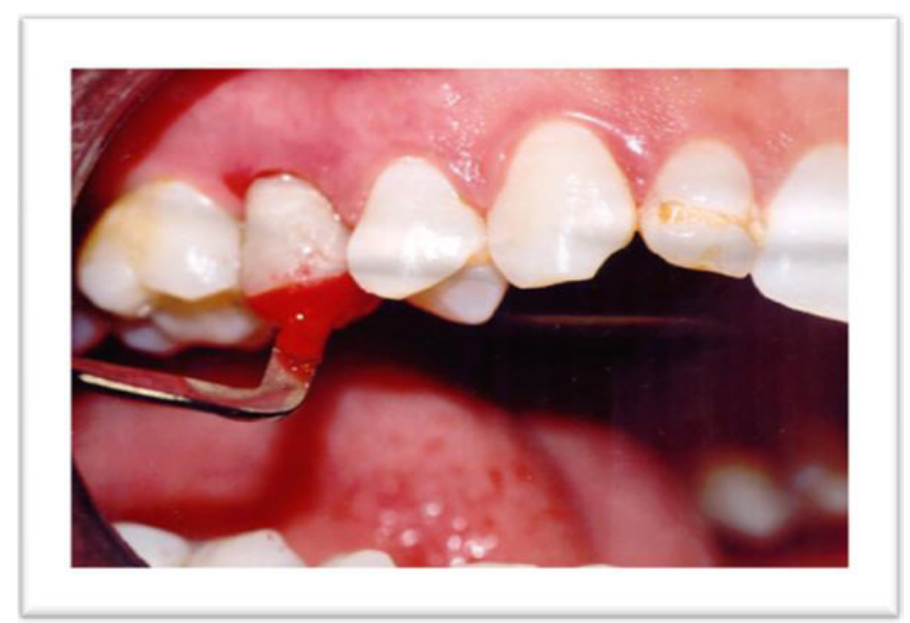

Figure 2: A layer of resin (Duralay) applied on occlusal surface

The patient was instructed to close the mouth in the maximum intercuspation position (MIP) and then perform right lateral, left lateral, and protrusive movements in succession, ending in the maximum intercuspation position (Figure 3, 4 and 5).

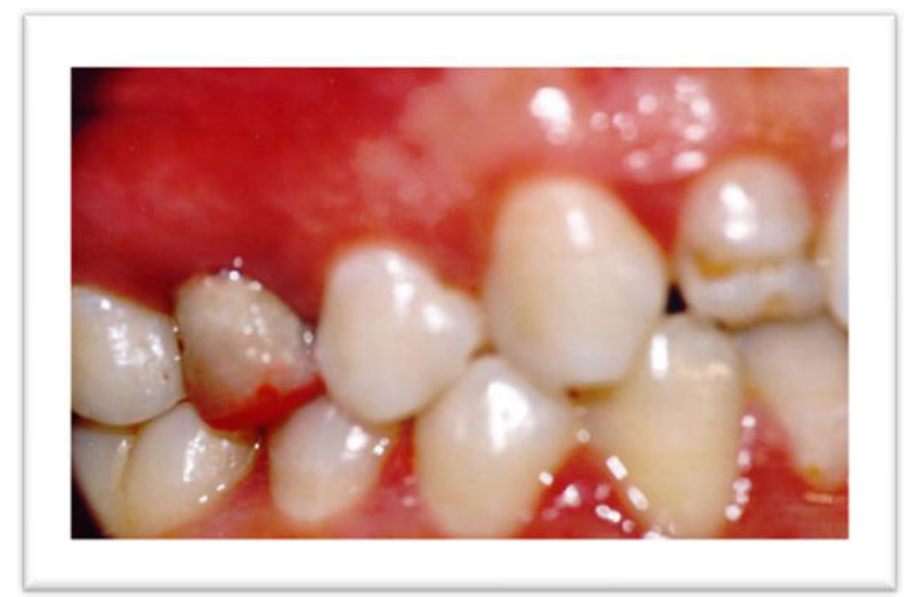

Figure 3: The patient closed in maximum intercuspation position (MIP) 
The paths of the cusps in working excursion are recorded and the area and direction of this excursive movement are demonstrated by fine lines in the inset. The path of the cusps in the non-working excursion are recorded next. The excess pattern resin was trimmed off using an acrylic trimmer. The patient was instructed to glide the mandible through all possible excursive movements to ensure capturing all border movements [16].

The paths of the cusps in working excursion were recorded and the area and direction of this excursive movement were demonstrated by fine lines in the inset. The path of the cusps in the non-working excursion were recorded next (Figure 6). The excess pattern resin was trimmed off using an acrylic trimmer.

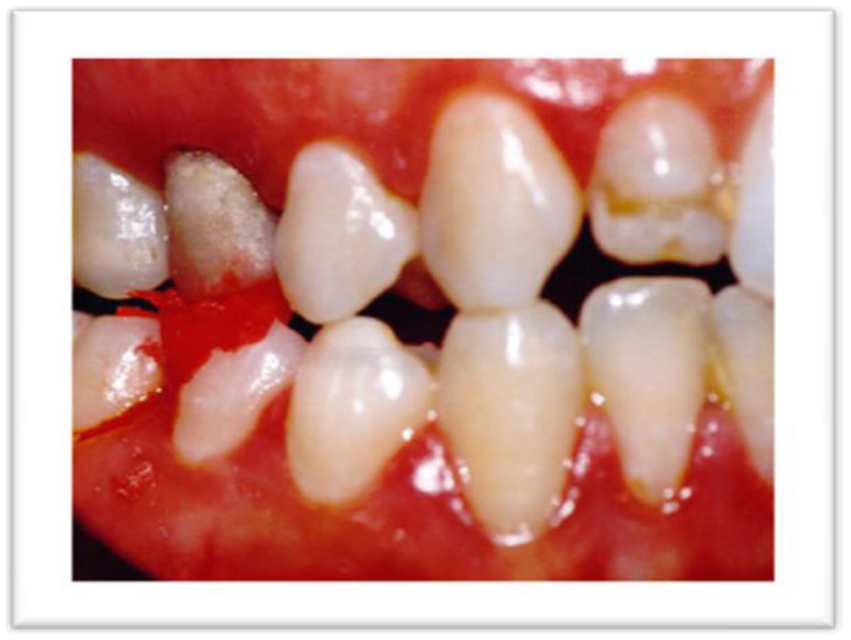

Figure 4: Lateral paths are recorded in working excursion.

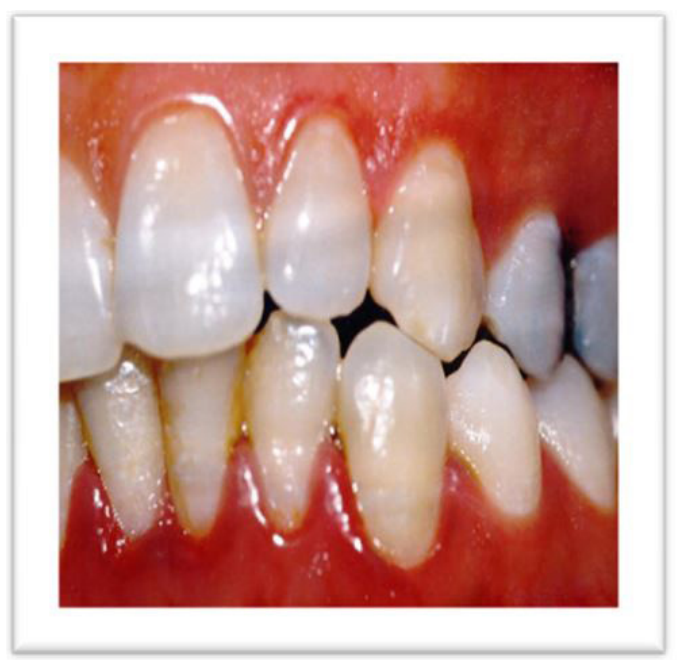

Figure 5: Lateral paths are recorded in nonworking excursion

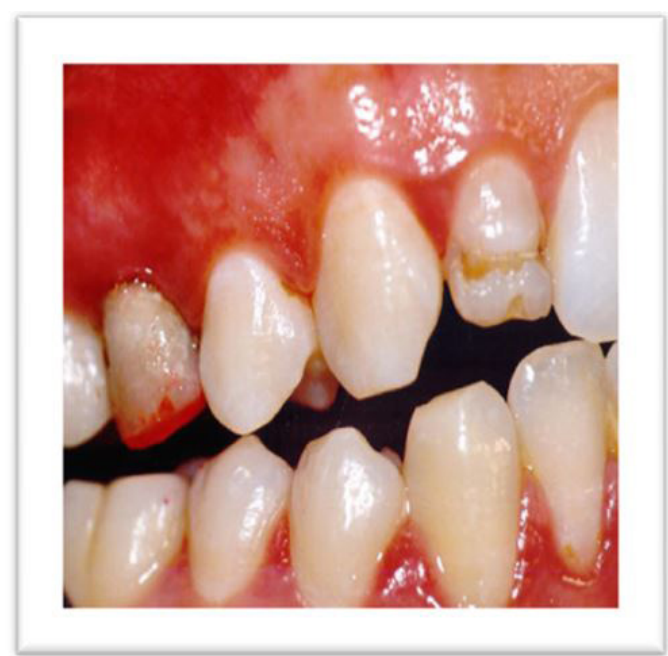

Figure 6: Protrusive paths are recorded last

(Figures 4, 5 and 6): The patient was instructed to perform the eccentric movements

The occlusal surface was examined and all eccentric contacts became visible with the wiping away of the resin (Figure 7) [17].

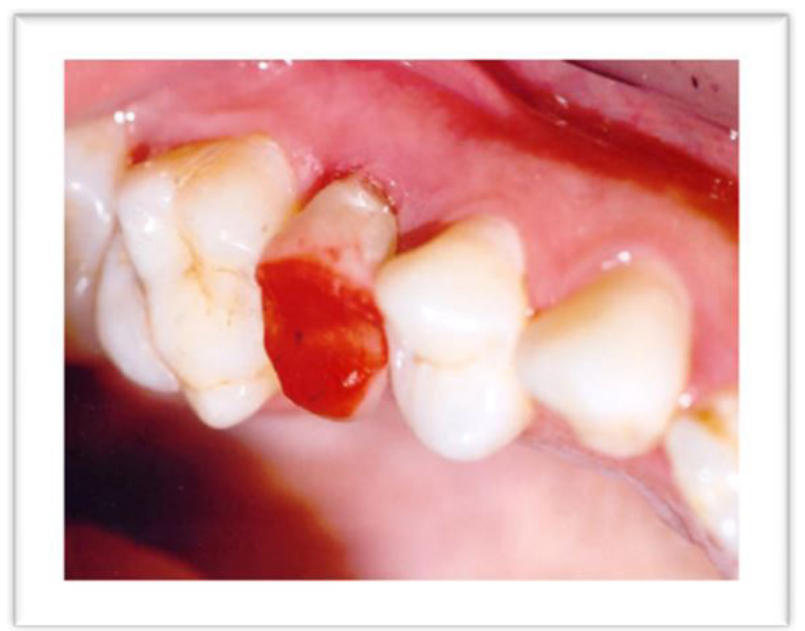

Figure 7: All occlusal contacts are high lightened and checked 
To obtain the functional core we used a simple artificial teeth support plate (denture tooth cards) as a $<<$ tray like $>>$ which was loaded with creamy fast setting stone (snow white of Kerr). It was positioned on the occlusal surface of the quadrant so that it covered at least one tooth at the both sides of the prepared premolar (Figure 8).

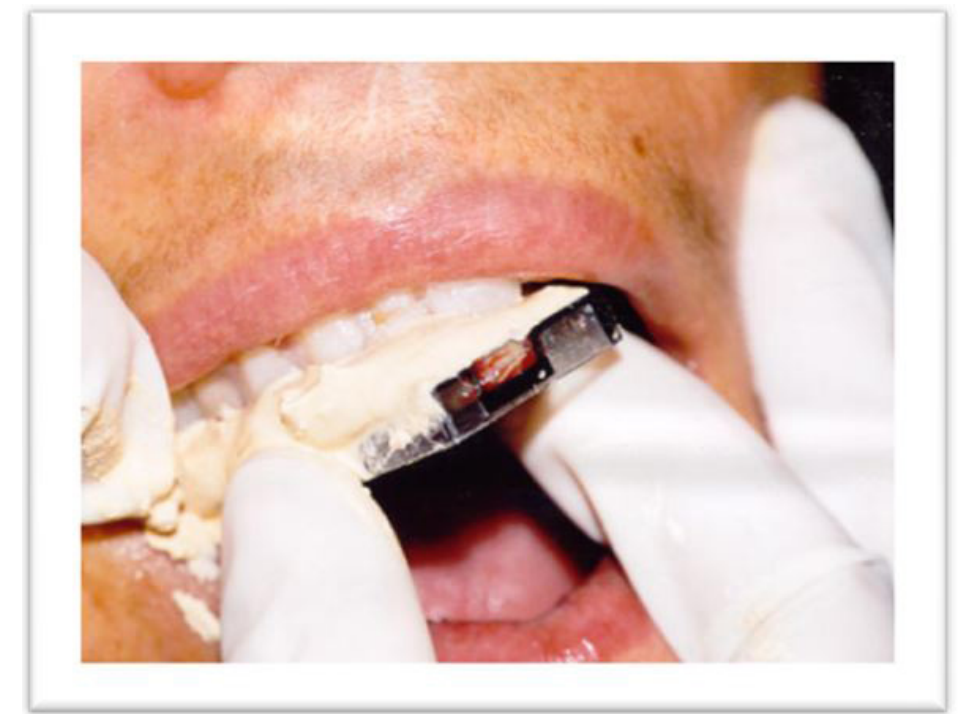

Figure 8: Creamy stone was brushed and poured into the resin record and denture tooth cards to record paths

The functional core is the stone replica of the movement of the cusps tips was removed and carefully checked and carefully checked (Figure 9).

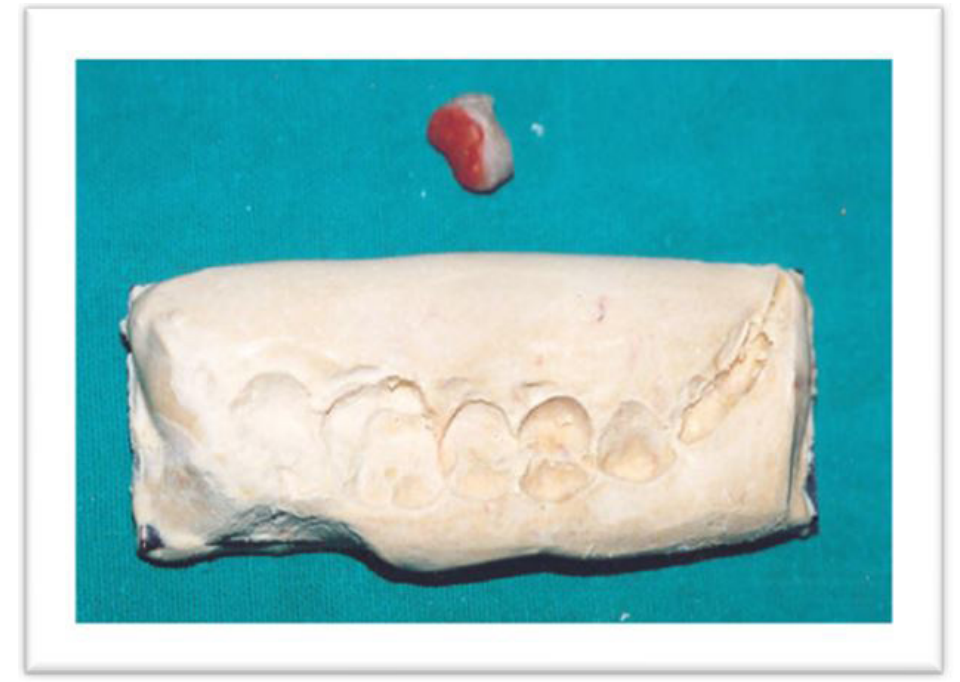

Figure 9: Functional core with temporary crown

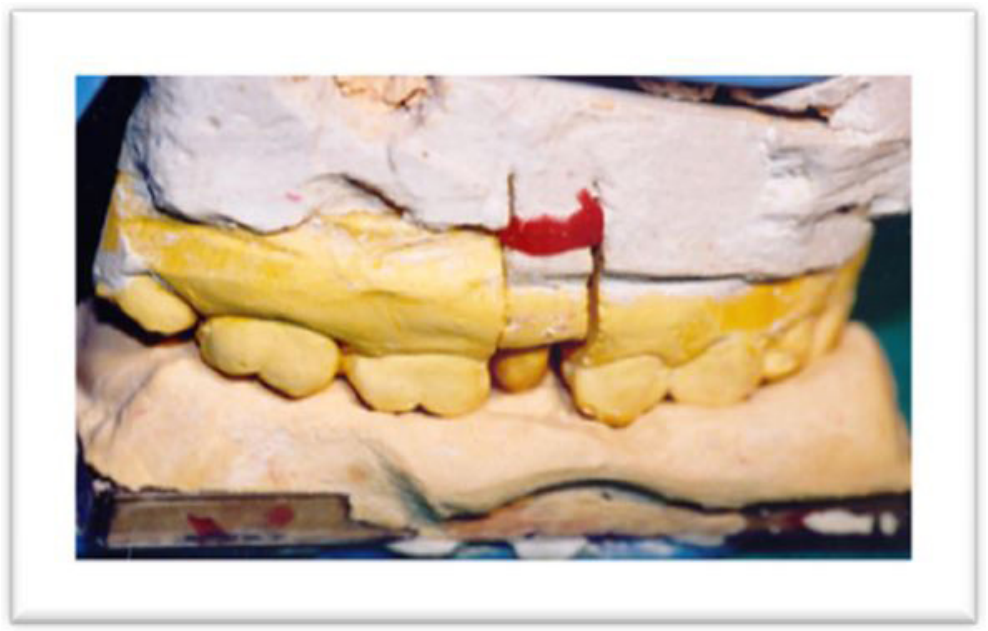

Figure 10: Mounting of the working cast and the functional core 


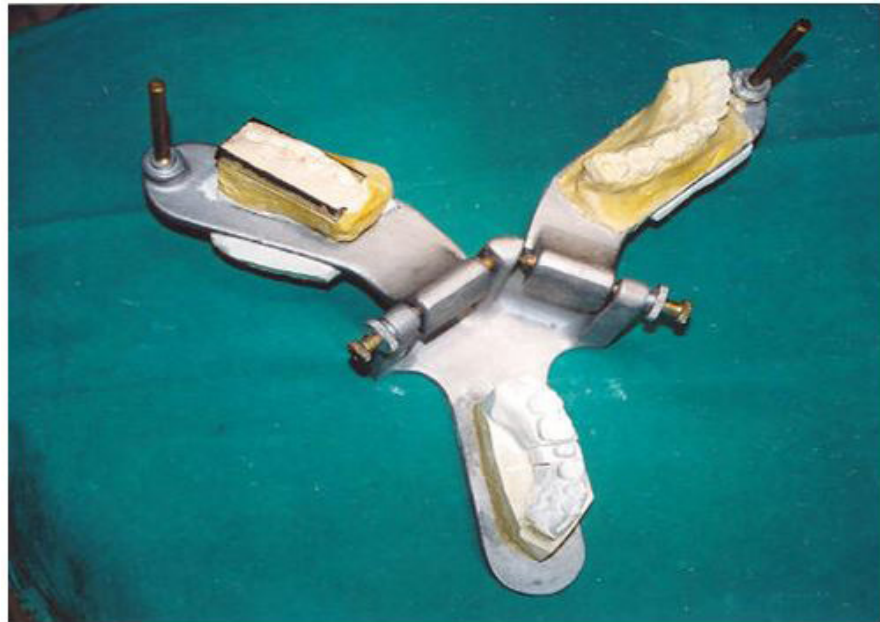

Figure 11: Twin Stage occluder of Hanau is most specifically designed for FGP technique

The functional cast (cast with the prepared tooth) was mounted with the upper member of a simple plastic occlude instead of twin stage of Hanau or a verticulator (Figures 10 and 11). Nevertheless the functional core cast was mounted on the lower one.

These paths registered with a stone matrix will be used by the ceramist as a guide and the framework was casted in a Nickel -chromium alloy following standard laboratory procedures. It was finished and polished, returned to the master cast, and tried intraorally. It always requires a try-in step to confirm fit, insertion retention, marginal integrity, and mostly the space left for cosmetic ceramic (Figure 12) [18,19].

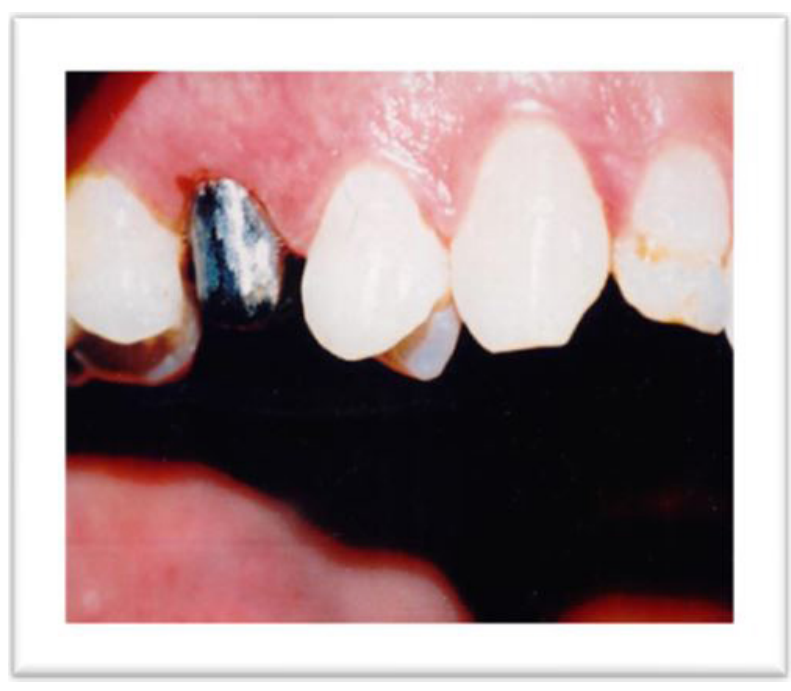

Figure 12: Trying metal framework

After shade selection the stratification of ceramics was performed with accurate checking of the occlusal morphology using the functional core (Figure 13).

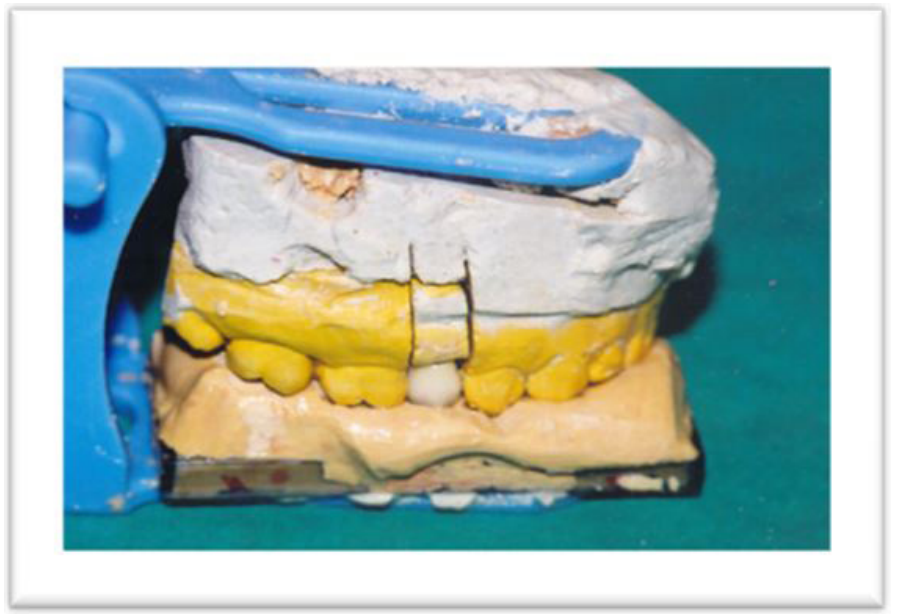

Figure 13: Ceramic stratification fitted to the die 
In the clinic, the metal ceramic crown was tried to verify static and functional occlusion using an articulating paper and with thickness 40 Microns. It can highlight any existing masticatory pressure interference clearly and can avoid unnecessary grinding of veneering material [20].

It was observed that there was absolutely no occlusal error identified in the MIP position. Only minimal eccentric interferences were identified and could be eliminated by selective grinding of ceramic. The final prosthesis was cemented using glass ionomer cement (GC Fuji II, GC Corporation, Japan) (Figure 14) [17].

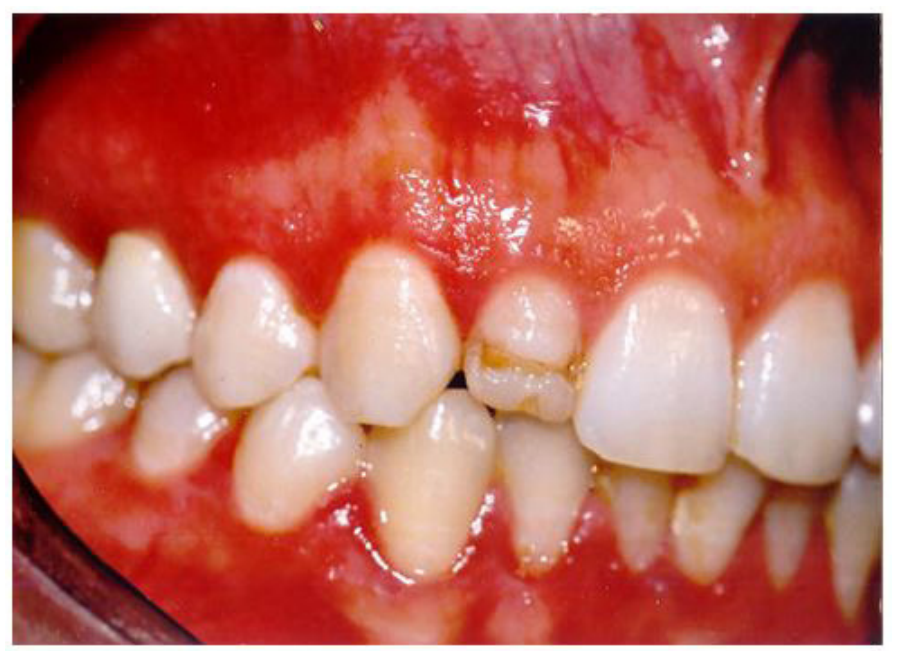

Figure14: Extra-oral View after Cementation

\section{Discussion}

Meyer suggested that 'no occlusal adjustment in the mouth should be necessary if the technique is carried out correctly [21]. Static and dynamic occlusal interference frequently needs to be corrected by selective grinding of the occlusal surface of conventional cast and ceramic-fused-to-metal restorations.

When understood and appreciated, use of the FGP technique is a straightforward and practical method to achieve harmonious occlusal anatomy of restorations with the anterior determinant/anterior guidance, the posterior determinant/condylar guidance, existing occlusal and cuspal anatomy, and the neuromuscular system [22].

Functionally generated path technique utilizes the patient's masticatory system to develop occlusion. The FGP still remains a technique that is simple, reliable, and unsurpassed in accuracy, but if the patient cannot control his neuromuscular movement, he may jeopardize the record of FGP $[23,24]$.

There are certain prerequisites for the use of FGP for the restoration of missing teeth, these are as follows:

- Presence of an optimal occlusion.

- Appropriate anterior guidance.

- Elimination of posterior interferences prior to making the restoration.

- The operator needs to have a good knowledge of occlusion and of mandibular movements; otherwise it may lead to an incomplete generation of FGP.

- No missing or broken down opposing teeth. Badly rotated, carious or poorly restored teeth in the opposing arch will not provide the occlusal pathways needed for shaping the occlusal surface and hence FGP should be avoided in these cases [14,15].

We used a temporary crown as support of resin Duralay which is also a support for FGP record and a simple affordable plastic occluder rather than a twin stage occluder or a verticalator.

This technique, comparable to the conventional FGP, permits achievement of an accurate occlusion by eliminating the inherent errors of the conventional method [17].

The occlusal morphology of the final prosthesis will be the same as the provisional prosthesis obtained from the registration of opposing teeth.

The time taken for this technique was drastically reduced to $5 \mathrm{~min}$. The patient reported absolute comfort with the prosthesis, this may be because the occlusal morphology was being generated directly in the patient's dentition path and can duplicate the whole stomatognathic system (condylar guidance and incisal guidance).

Furthermore, the size of articulation paper mark is not an accurate indicator to employ in the selection of tooth contacts for occlusal adjustment treatment. 
However, employing a non-subjective, quantifying occlusal indicator like computerized occlusal analysis (T-Scan), to guide the selection of tooth contacts for occlusal adjustment treatment, may produce more comprehensive and evidence-based results [25].

On the another hand, the modified generated path technique, compared to the conventional one, when learned to students in our faculty of dentistry, it has been easily understood.

The modified FGP as the conventional indirect method includes numerous steps, including impression making, occlusal registration, cast mounting, and so forth. Each step in the sequence of this method has inherent errors, that affect the accuracy of occlusion and they are as follow [26]

- Deformation of impression,

- Distortion of occlusal registration material,

- Incorrect mounting of a cast to an articulator,

- Poor fit of cast prosthesis to an abutment and

- Improper occlusion of the prosthesis.

- Some differences between occlusal details of the ideal anatomical configuration while the surface is functionally ideal [27].

Minimal occlusal adjustments are required during the clinical try-in stage due to difference of fitting between the temporary crown and the metal ceramic crown and somewhat caused by the physiologic mobility of the teeth.

As with any technique, the FGP technique also has certain disadvantages. Some of the limitations of this technique are related to the operator and other are related to the patient as follows:

- Operator lacking knowledge of occlusion and of mandibular movements

- Uselessness of this technique in cases with short clinical crowns and unfavorable occlusal morphology of the opposing teeth

- Uselessness in patients having attired teeth.

- Patients lacking proper neuromuscular control cannot be selected for this technique.

- In patients having disharmony in occlusion (malocclusions like deep bite and cross bite) and temporomandibular joint dysfunction.

- Good laboratory support is a basic requirement, without which successful results are difficult to achieve [17].

\section{Conclusion}

The technique of FGP finds a place in the arsenal of methods for recording the occlusion in fixed prostheses. The success of functionally generated path technique requires the cooperation of 3 actors; the patient, the operator and the laboratory.

Nevertheless, multicenter clinical trials with long follow-up periods should be conducted to confirm the results obtained in present case report, and to compare 2 crowns one made with the traditional method and the other one with the FGP modified technique on the same tooth to analyze the differences [17].

\section{Acknowledgement}

Special Thanks to Mr. Hassen Rached for the revision of the English grammar and syntax errors.

\section{References}

1. Meyer FS (1943) A new, simple and accurate technique for obtaining balanced and functional occlusion. J Am Dent Assoc 21: 195-203.

2. Meyer FS (1959) The generated path technique in reconstruction dentistry. Part I. Complete dentures. J Prosthet Dent 9: $354-66$.

3. Meyer FS (1959) The generated path technique in reconstruction dentistry. Part II. Fixed partial dentures. J Prosthet Dent 9: 432-40.

4. Mann AW, Pankey LD (1960) Oral rehabilitation. Part I. Use of the P-M Instrument in treatment planning and in restoring the lower posterior teeth. J Prosthet Dent 10: 135-50.

5. Pankey LD, Mann AW (1960) Oral rehabilitation. Part II: Reconstruction of the upper teeth using a functionally generated path technique. J Prosthet Dent 10: 151-62.

6. Mann AW, Pankey LD (1959) Oral rehabilitation utilizing the Pankey-Mann Instrument and a functional bite technique. Dent Clin North Am 3: 215-30.

7. Mann AW, Pankey LD (1963) Concepts of occlusion. The P.M. Philosophy of Occlusal Rehabilitation. Dent Clin North Am 7: 621-36.

8. Meyer FS (1933) A new, simple and accurate technique for obtaining balanced and functional occlusion. JADA 21: 195-203.

9. Olthoff LW, Van Der Zel JM, De Ruiter WJ, Vlaar ST, Bosmann F (2000) Computer modeling of occlusal surfaces of posterior teeth. J Prosthet Dent 84: 154-62.

10. Zimmermann EM (1966) Modifications of functionally generated path procedures. J Prosthet Dent 16: 1119-26.

11. The glossary of prosthodontic terms (2005) J Prosthet Dent 94: 10-92.

12. Dawson PE (1974) Evaluation, Diagnosis and Treatment of Occlusal Problems (1 $1^{\text {st }}$ edn) CV Mosby, Canada.

13. Wassell RW, Walls AW, Steele JG (2002) Crowns and extra-coronal restorations: materials selection. Br Dent J 192: 199-202, 205-11. 
14. Shillingburg HT, Hobo S, Whitsett LD, Jacobi R, Brackett SE (1997) Fundamentals of fixed prosthodontics. ( $3^{\text {rd }}$ edn) Quintessence Publishing Co. Inc, United States.

15. Dawson PE (1989) Evaluation, diagnosis and treatment of occlusal problems (2nd edn) The C.V. Mosby Company, St.Louis, Baltimore, Toronto, Canada. 16. Patil PG, Nimbalkar-Patil SP, Kulkarni RS (2015) Functionally generated pathways to develop occlusal scheme for removable partial denture. J interdisci dent 5: 154-7.

17. Prashanti E, Sajjan S, Reddy JM (2009) Fabrication of fixed partial dentures using functionally generated path technique and double casting. Indian J Dent Res 20: $492-5$.

18. Curtis SR (1999) Functionally generated paths for ceramometal restorations. J Prosthet Dent 81: 33-6.

19. Saafi J, Debbabi I, Chakroun M, Nouira Z, Harzallah BH, et al. (2017) Esthetic Restoration of Two Severely Decayed Maxillary Central Incisors: A Case Report. J Oral Health Dent Sci 1: 104.

20. Brizuela-Velasco A, Álvarez-Arenal Á, Ellakuria-Echevarria J, del Río-Highsmith J, Santamaría-Arrieta G, Martín-Blanco N (2015) Influence of Articulating Paper Thickness on Occlusal Contacts Registration: A Preliminary Report. Int J Prosthodont 28: 360-2.

21. Kafandaris NM (1981) A modified functionally generated path technique for making maxillary posterior ceramometal restorations. J Prosthet Dent 46: 29-31. 22. Du Vall NB, Rogers PM (2013) Application of the Functionally Generated Path Technique to Restore Mandibular Molars in Bilateral Group Function Occlusion. J Prosthodont 22: 226-32.

23. Manary DG, Holland GA (1984) Evaluation of mandibular movement recording and programming procedures for a molded condylar control articulator system. J Prosthet Dent 52: 275-80.

24. Winstanley RB (1977) Observations on the use of the denar pantograph and articulator. J Prosthet Dent 38: 660-72.

25. Qadeer S, Kerstein R, Yung Kim RJ, Huh JB, Shin SW (2012) Relationship between articulation paper mark size and percentage of force measured with computerized occlusal analysis. J Adv Prosthodont 4: 7-12.

26. Matsushita K (1982) A study on occlusal accuracy of cast crown - the changes of occlusal height of full cast crown by the fabrication steps. J Jpn Prosthodont Soc 26: 250-66.

27. Minagi S, Tanaka T, Sato T, Matsunaga T (1998) Double-casting method for fixed prosthodontics with functionally generated path. J Prosthet Dent 79: 120-4. 